\title{
SLE-Related Longitudinal Myelitis with Favorable Outcome
}

\author{
Marco Mula, Laura Bolamperti, Claudia Varrasi, Daniela Mittino, Silvia Colombi, \\ Alessandro Stecco, Roberto Cantello, Francesco Monaco
}

Can. J. Neurol. Sci. 2009; 36: 393-397

Systemic lupus erythematosus (SLE) is an autoimmune disease with multi-organ involvement, defined by its clinical features and the presence in the blood of antibodies against one or more components of cell nuclei ${ }^{1}$. Neurological and psychiatric complications of SLE include seizures, psychosis, cerebrovascular disease, cognitive dysfunction ${ }^{2}$ and headache ${ }^{3}$, described in up to $75 \%$ of patients ${ }^{4,5}$. In general terms, any part of the nervous system ${ }^{6,7}$ may be involved, however, central nervous system (CNS) complications are of relevance because can significantly affect prognosis and mortality.

Different pathogenetic mechanisms have been claimed to play a relevant role in CNS complications of SLE such as vasculitis, antibodies against brain tissues, deposition of immunocomplex, overproduction of cytokines, thrombosis, and hemorrhage ${ }^{8}$.

Myelopathy is one of 19 possible syndromes described by the American College of Rheumatology ${ }^{9}$, with transverse myelitis (TM) being a rare but serious neurologic complication. It presents as a rapidly progressive motor, sensory and autonomic dysfunction $^{10-12}$, with uncertain pathophysiology, whose prevalence seems to be in the region of $1-2 \%$ among SLE patients ${ }^{10,13-16}$.

Since the initial description of the first nine cases in $1968^{17}$, SLE-related TM is now a well-known clinical entity and a number of authors pointed out the close relationships with antiphospholipid antibodies (aPL) syndrome. In fact, from $55 \%$ to $64 \%$ of SLE-related TM present high levels of aPL, which is higher than that reported in the general SLE population (30$50 \%)^{18-19}$. Different authors, therefore, speculated that the pathogenesis of TM may be related to a vascular thrombosis or to a direct interaction between aPL and membrane phospholipids at the spinal cord level ${ }^{14}$.

A rare variant of the SLE-related myelopathy is longitudinal myelitis (LM), which is defined by the continuous involvement of more than four spinal cord segments ${ }^{20-23}$. In fact, one to four spinal segments are generally involved in SLE-related TM ${ }^{20,23}$. Longitudinal myelitis needs to be promptly diagnosed and recognized because it is characterized by an acute catastrophic onset and an unfavorable prognosis, and a closer association with aPL syndrome has been suggested when compared to $\mathrm{TM}^{20}$.

Here, we describe a patient presenting with LM and peripheral neuropathy as early manifestations of SLE, with negative aPL and a favorable outcome. Clinical, electrophysiological and radiological features at the onset and during the follow-up are discussed.

\section{Case Report}

\section{Patient history}

A 63-year-old woman was admitted to our Department for acute/sub-acute onset of fever, severe neck pain, weakness predominantly affecting both legs and the left arm, left-sided tingling sensations and sphincter disturbances (acute urinary retention, ileus paralyticus). The neurological examination revealed paraparesis and left arm paresis with dystonic posturing of the left hand and left foot. Reflexes were brisk at the four limbs and prevalent at the left side. She presented a bilateral Babinski. All-sensory modalities were lost below D6 level. Consciousness was preserved and the mental state was normal. Cranial nerves were normal.

The patient had a childhood history of rheumatic fever and an eight-year history of bilateral glaucoma. Since the age of 40 she referred migratory arthralgias involving both hips and ankles successfully treated with steroids (no more information was available). Since the age of 60 she referred with skin lesions, not better specified which were treated with topical steroids. There was no history of face rush, Reynaud's phenomenon or oral ulcers. There was no history of neurological or neuromuscular disorders.

\section{Laboratory work-up}

Routine blood tests including inflammation indexes, electrolytes, renal, liver and thyroid function, creatine kinase, glucose, vitamin B12, folic acid, hemoglobin level, platelet count, white blood cell count, prothrombin time and activated partial prothrombin time were all within the normal range.

Rheumatologic blood tests showed highly positive antinuclear antibodies (4.91 IU/mL, normal range 0-1 IU/mL) speckled pattern; high levels of anti-dsDNA antibodies $(69.4 \mathrm{IU} / \mathrm{mL}$, normal range 0-30 IU/mL); dRVTT screening $38.7 \mathrm{sec}$ (normal

From the Division of Neurology (MM, LB, CV, DM, RC, FM), Division of Internal Medicine (SC), Division of Radiology (AS), Maggiore Hospital, Amedeo Avogadro University, Novara, Italy.

Received October 16, 2008. Final Revisions Submitted December 11, 2008 Correspondence to: Marco Mula, Division of Neurology, Amedeo Avogadro University, C.so Mazzini, 18, 28100 Novara, Italy. 


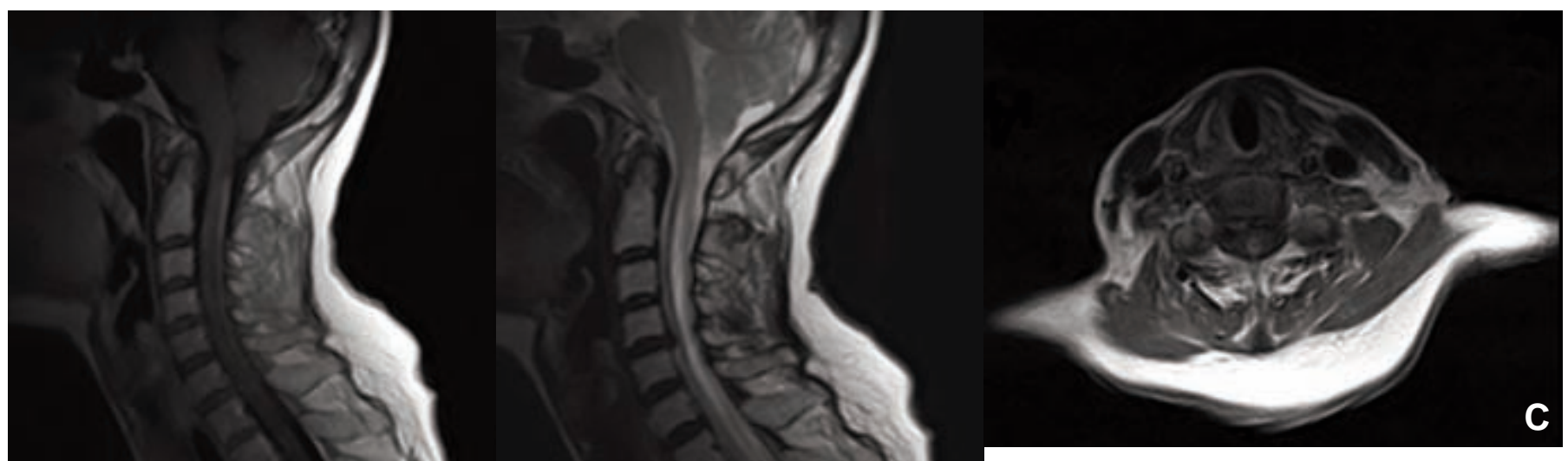

Figure: (A) Admission scan, sagittal plan, T1weighted image after Gadolinum showing peripherical enhancement; (B) Admission scan, sagittal plan, T2-weighted image showing diffuse centromedullar hyperintensity from C2 to T3 level with spinal cord swelling; (C) Admission scan, axial plan, Spine Echo T1-weighted image showing intense centromedullary band-like enhancement; some artifacts from movements are visible; (D) Four-month follow up scan, sagittal plan, Tlw image after Gadolinum showing no more swelling or contrast enhancement; $(E)$ Four-month follow-up scan, sagittal plan, T2w image showing no more swelling, a gliotic scar extending cranio-caudally along the spinal cord is still recognizable.

range 0-38 sec); dRVVT ration 1.33 (normal range 0.80-1.30); serum complement $\mathrm{C} 3$ and $\mathrm{C} 4$ levels were slightly reduced (C3 $=85 \mathrm{mg} / \mathrm{dL}$, normal $90-180 \mathrm{mg} / \mathrm{dL} ; \mathrm{C} 4=9 \mathrm{mg} / \mathrm{dl}$, normal range 10-40 mg/dl); ENA (anti-Sm, Sm/RNP, SSA, SSB, JO1, SCL-70 and ENA screening) were negative; aPL $(\mathrm{IgG}=1.1 \mathrm{U} / \mathrm{ml}, \mathrm{IgM}$ 1.3 $\mathrm{U} / \mathrm{ml}$ ) and anticardiolipin antibodies (GPL 1.3 U/ml, MPL $1.6 \mathrm{U} / \mathrm{ml}$ ) were normal. Lupus anticoagulant (PTT-LA), Protein $\mathrm{C}$, Rheuma test, W-Rose test and immunoglobulin levels (IgG, $\operatorname{Ig} \mathrm{A}, \operatorname{IgM})$ were in the normal range.

A lumbar puncture revealed normal white cells count with an elevated protein level of $110 \mathrm{mg} / \mathrm{dL}$ (normal range 15-45 $\mathrm{mg} / \mathrm{dL}$ ). Glucose and chlorides levels were normal, same oligoclonal pattern in serum and cerebrospinal fluid (CSF) suggesting no intrathecal synthesis. S-IgG $1040 \mathrm{mg} / \mathrm{dL}$, LCRIgG $4.54 \mathrm{mg} / \mathrm{dl}(0,80-3,80)$; IgG index 0.82 (normal range < 0,70); Tourtellotte Index 6.39 (<3.30). PCR for HSV1, HSV2 and Adenovirus were negative. HIV serology was negative.

\section{MRI study}

Magnetic resonance (MR) scan was performed on a Signa Contour (General Electric, Milwaukee, IL, USA) 0.5 Tesla 
magnet, with updated gradients. The protocol included the following pulse sequences: 1) Fast Spin Echo (SE) T2 weighted (w)(TR 2340, TE 110, ETL 8, 4 mm thickness, 2 nex, matrix 256x256) and 2) SE T1w before and after Gadolinum injection (TR 440, TE 11, 4 mm thickness, 1 nex, matrix 256x256), 3) STIR (TR 3000, TE 20, TI 100, ETL 10, 4 mm thickness, 2 nex, matrix $256 \times 256$ ) all acquired in the sagittal plane, with a $30 \mathrm{~cm}$ Field of View (FOV). The SE T1w pulse was acquired also in the axial plane (TR 340, TE 23, $4 \mathrm{~mm}$ thickness, 1 nex, matrix 256x256) after Gadolinium injection to better identify the exact spinal cord area involved.

Brain MRI was normal. Spinal cord MRI revealed longitudinal decreased signal intensity in T1-weighted images (WI) and increased signal intensity in T2 WI from C1 to D10 levels with cord swelling. There was no gadolinium contrast enhancement (Figure).

\section{Neurophysiologic study}

Nerve conduction study showed a severe motor axonal neuropathy involving the lower extremities. M wave was not evoked during nerve conduction study on bilateral peroneal nerves. Bilateral median nerve conduction was within the normal range. Right ulnar nerve conduction was slightly reduced.

\section{Clinical course}

According to the laboratory data, nerve conduction study and MRI findings, the patient was diagnosed as having SLE-related acute LM and polyneuropathy. She received plasmapheresis (three sessions per week over the course of two weeks) and subsequently she started cyclophosphamide (CYC) $(15 \mathrm{mg} / \mathrm{kg}$, total $1 \mathrm{~g}$. per application) and prednisolone (100 $\mathrm{mg}$ per application) intravenous pulse treatment monthly over the course of four months. During this period, the patient was maintained with daily oral prednisone $(25 \mathrm{mg})$.

Four-month follow-up showed complete recovery of the sensory deficits, significant improvement in motor function and occasional urinary urgency. Four-month spinal cord MRI followup showed a slight improvement with no more swelling or contrast enhancement. Nerve conduction study was unchanged. The two-year follow-up showed complete recovery (the patient was able to walk autonomously), there were no sphincter disturbances or sensory problems. Nerve conduction study showed significant improvement with mild motor axonal neuropathy involving only the lower extremities. Blood tests were normal.

\section{Discussion}

It has been estimated that TM can be the early manifestation of SLE in $39 \%$ of cases $^{15,24}$ but $42 \%$ of patients with SLE can present with TM during the first five years ${ }^{14-15,25}$, suggesting the spinal cord involvement is not rarely reported in patients with SLE.

Although TM is a well-known complication of SLE, the interest in LM is recent with the description by Deodhar et $\mathrm{al}^{20}$ of a patient with continuous involvement of the spinal cord from $\mathrm{C} 3$ to $\mathrm{T} 2$ levels and from $\mathrm{T} 7$ to the conus medullaris. Subsequently, Téllez-Zenteno and collaborators ${ }^{21}$ presented the first series of SLE-related LM.
A recent review of 12 cases by Heinlein and Gertner ${ }^{26}$ revealed that LM is generally associated with SLE but it may occur also as a complication of other autoimmune diseases such as Sjiogren's syndrome.

Pathogenic mechanisms of SLE-related myelitis (both TM and LM) is still unclear, although it has been speculated that immune-complex mediated vasculitis, antineuronal antibodies, white matter degeneration ${ }^{27}$ and hypercoagulability from aPL may play a role ${ }^{8,28}$. Kimura Kato and colleagues ${ }^{7}$ commented $^{-}$ that mechanisms other than those involved in SLE (e.g. concomitant thrombosis or a delay in starting treatment) may be implicated. In particular, LM seems to be often related to aPL either as vascular occlusive phenomena of the spinal cord mediated by aPL or as a direct interaction between aPL and spinal cord phospholipids ${ }^{13,21,29}$. In fact, some authors suggested that all patients with LM should be tested not only for SLE but also for $\mathrm{aPL}^{13,30}$.

The outcome of SLE-related TM is generally favorable, with a complete recovery in $50 \%$ of patients, a partial recovery in $29 \%$ and no improvement in $21 \%{ }^{15}$. On the contrary, at least $50 \%$ of patients with LM seem to have a poor prognosis ${ }^{26}$. Although the final prognosis relies on different variables such as a prompt diagnosis, specific treatment strategies and the extent of the neurological involvement ${ }^{31}$, previous reports showed that a rapid diagnosis and an early aggressive immunosuppressive therapy using high doses of corticosteroids and pulsed IV-CYC is associated with satisfactory outcome only in $\mathrm{TM}^{25,32-33}$, being still controversial for LM due to the paucity of published studies. Téllez-Zenteno et $\mathrm{al}^{21}$ commented that the outcome can be unfavorable in most cases even if an aggressive treatment with high dose corticosteroids and CYC is started. In fact, this drugcombination showed to be successful in only one reported case $\mathrm{e}^{20}$ and a slow improvement was noted in another one out of the six described by Téllez-Zenten ${ }^{21}$.

Our patient showed a very good response to treatment and a final favorable outcome. Compared to other reported cases, the main difference relates to the combined use of synchronized plasmapheresis. Despite the paucity of data on therapeutic interventions in LM, it is plausible to speculate that early aggressive treatment strategies, using a combination of plasmapheresis and immunosuppressive agents, need to be considered in patients with LM. This is further supported by a few other cases characterized by a favorable outcome adopting early aggressive treatment strategies. Moranne et $\mathrm{al}^{34}$ described the first report of LM, in a pregnant patient with SLE, promptly treated with corticosteroid and plasmapheresis with complete recovery. Lehnhardt et $\mathrm{al}^{35}$ described a case of LM and SLErelated aseptic meningitis in which only autologous hematopoietic stem cell transplantation was successful with no evidence for residual serological or neuroradiological activity of SLE. Mok et $\mathrm{al}^{36}$ reported the preliminary experience of the use of combined corticosteroid and mycophenolate mofetil, a relatively new immunosuppressive agent, in the treatment of SLE-related myelopathy, concluding that immunosuppressive treatments should be considered in some selected cases, especially if refractory to $\mathrm{CYC}^{37-38}$.

Nonetheless, it is also tempting to speculate that the lack of aPL in our patient may represent an index of favorable prognosis. It has been suggested that patients with LM should always be 
tested for aPL ${ }^{21}$, it being possibly implicated in the pathogenetic processes of the LM itself. Further research is needed to clarify whether aPL may represent an index of poor response to treatment in patients with LM.

As to the peripheral nervous system (PNS) involvement in SLE patients, a Guillain-Barré syndrome, an autonomic neuropathy, mononeuropathies, plexopathies, and a sensorimotor polyneuropathy were reported ${ }^{9}$. The PNS involvement is usually regarded as a rare complication of SLE, with prevalence rates ranging between $3 \%$ and $27 \%$ in various clinical series ${ }^{39-43}$. The neuropathic process is usually modestly progressive over the time, with possible fluctuations and not necessarily irreversible $\mathrm{u}^{44-45}$. To the best of our knowledge, there is only one report of simultaneous SLE-related LM and peripheral neuropathy ${ }^{7}$. In our patient, the favorable outcome was also found for PNS involvement with nerve conduction study followup demonstrating a progressive improvement over two years. In our opinion, these findings further support the hypothesis that patients with negative aPL may configure a particular clinical phenotype with a favorable prognosis.

In conclusion, LM represents a rare but serious and possibly life-threatening complication of SLE. Data on therapeutic strategies are still scanty as the identification of clinical and biological indexes for prognosis and response to treatment. We described a case of LM with associated polyneuropathy without aPL which displayed a favorable outcome after aggressive immune-suppressive treatment. Further studies involving larger samples are warranted to develop standardized clinical protocols for therapy and prognosis.

\section{REFERENCES}

1. Mills JA. Systemic lupus erythematosus. N Engl J Med. 1994;330:1871-9.

2. Hay EM, Black D, Huddy A, Creed F, Tomenson B, Bernstein RM, et al. Psychiatric disorder and cognitive impairment in systemic lupus erythematosus. Arthritis Rheum. 1992;35:411-6.

3. Isenberg DA, Meyrick-Thomas D, Snaith ML, McKeran RO, Royston JP. A study of migraine in systemic lupus erythematosus. Ann Rheum Dis. 1982;41:30-2.

4. Afeltra A, Garzia P, Mitterhofer AP, Vadacca M, Galluzzo S, Del Porto F, et al. Neuropsychiatric lupus syndromes: relationship with antiphospholipid antibodies. Neurology. 2003;61:108-10.

5. Sanna G, Bertolaccini ML, Mathieu A. Central nervous system lupus: a clinical approach to therapy. Lupus. 2003;12:935-42.

6. Sibley JT, Olszynski WP, Decoteau WE, Sundaram MB. The incidence and prognosis of central nervous system disease in systemic lupus erythematosus. J Rheumatol. 1992;19:47-52.

7. Kimura Kato Y, Seino Y, Hirayama Y, Aramaki T, Yamaguchi H, Amano H, et al. Systemic lupus erythematosus related transverse myelitis presenting longitudinal involvement of the spinal cord. Intern Med. 2002;41:156-60.

8. Provenzale J, Bouldin TW. Lupus-related myelopathy: report of three cases and review of the literature. J Neurol Neurosurg Psychiatry. 1992;55:830-5.

9. ACR ad hoc committee neuropsychiatric lupus nomenclature. The American College of Rheumatology nomenclature and case definitions for neuropsychiatric lupus syndromes. Arthritis Rheum. 1999;42: 599-608.

10. Andrianakos AA, Duffy J, Suzuki M, Sharp JT. Transverse myelopathy in systemic lupus erythematosus. Report of three cases and review of the literature. Ann Intern Med. 1975;83: 616-24.

11. Ropper AH, Poskanzer DC. The prognosis of acute and subacute transverse myelopathy based on early signs and symptoms. Ann Neurol. 1978;4:51-9.
12. Cho BK, Song JS, Bae SK, Yoon SJ, Park W. A case of transverse myelitis as a first manifestation of systemic lupus erythematosus. J Korean Rheum Assoc. 2000;7:263-7.

13. Lavalle C, Pizarro S, Drenkard C, Sánchez-Guerrero J, AlarcónSegovia D. Transverse myelitis: a manifestation of systemic lupus erythematosus strongly associated with antiphospholipid antibodies. J Rheumatol. 1990;17:34-7.

14. West SG. Neuropsychiatric lupus. Rheum Dis Clin North Am. 1994;20:129-58.

15. Kovacs B, Laferty TL, Brent LH, DeHoratius RJ. Transverse myelopathy in systemic lupus erythematosus: an analysis of 14 cases and review of the literature. Ann Rheum Dis. 2000;59: $120-4$

16. Yazawa S, Kawasaki S, Ohi T, Shiomi K, Sugimoto S, Kawagoe J, et al. Development of severe longitudinal atrophy of thoracic spinal cord following lupus related myelitis. Intern Med J. 2001;40:353-7.

17. Penn AS, Rowan AJ. Myelopathy in systemic lupus erythematosus. Arch Neurol. 1968;18:337-49.

18. Alercon-Segovia D, Deleze M, Oria CV, Sanchez-Guerrero J, Gomez-Pacheo L, Cabiedes J, et al. Antiphospholipid antibodies and the antiphospholipid syndrome in systemic lupus erythematosus. A prospective analysis of 500 consecutive patients. Medicine (Baltimore). 1989;68:353-65.

19. Sanna G, Bertolaccini ML, Cuadrado MJ, Khamashta MA, Highes GRV. Central nervous system involvement in the antiphospholipid (Hughes) syndrome. Rheumatology. 2003;42: 200-13.

20. Deodhar AA, Hochenedel T, Bennett RM. Longitudinal involvement of the spinal cord in a patient with lupus related transverse myelitis. J Rheumatol. 1999;26:446-9.

21. Téllez-Zenteno JF, Remes-Troche JM, Negrete-Pulido RO, DavilaMaldonado L. Longitudinal myelitis associated with systemic lupus erythematosus: clinical features and magnetic resonance imaging of six cases. Lupus. 2001;10:851-6.

22. Chen HC, Lai JH, Juan CJ, Kuo SY, Chen CH, Chang DM. Longitudinal myelitis as an initial manifestation of systemic lupus erythematosus. Am J Med Sci. 2004;327:105-8.

23. Suzuki K. The role of immunoadsorption using dextran-sulfate cellulose columns in the treatment of systemic lupus erythematosus. Ther Apher. 2000;4:239-43.

24. Dittrich E, Schmaldienst S, Langer M, Jansen M, Hörl WH, Derfler $\mathrm{K}$. Immunoadsorption and plasma exchange in pregnancy. Kidney Blood Press Res. 2002;25:232-9.

25. Mok CC, Lau CS, Chan EY, Wong RW. Acute transverse myelopathy in systemic lupus erythematosus: clinical presentation, treatment and outcome. J Rheumatol. 1998;25: 467-73.

26. Heinlein AC, Gertner E. Marked inflammation in catastrophic longitudinal myelitis associated with systemic lupus erythematosus. Lupus. 2007;16: 823-6.

27. McCune WJ, Golbus J, Zeldes W, Bohlke P, Dunne R, Fox DA. Clinical and immunologic effects of monthly administration of intravenous cyclophosphamide in severe systemic lupus erythematosus. N Engl J Med. 1988;318:1423-31.

28. Theodoridou A, Settas L. Demyelization in rheumatic diseases. J Neurol Neurosurg Psychiatry. 2006;77:290-5.

29. Rheu CW, Lee SII, Yoo WH. A catastrophic-onset Longitudinal myeilitis accompanied by bilateral internuclear ophthalmplegia in a patient with systemic lupus erythematosus. J Korean Med Sci. 2005;20:1085-8.

30. Medina-Rodriguez F, Garcia E, Fraga A. Transverse myelitis and antiphospholipid antibodies. J Rheumatol. 1990;17:1250.

31. Habr F, Wu B. Acute transverse myelitis in systemic lupus erythematosus: a case of rapid diagnosis and complete recovery. Conn Med. 1998;62:387-90.

32. Barile L, Lavalle C. Transverse myelitis in systemic lupus erythematosus- the effect of IV pulse methylprednisolone and cyclophosphamide. J Rheumatol. 1992;19:370-2.

33. Harisdangkul V, Doorenbos D, Subramony SH. Lupus transverse myelopathy: better outcome with early recognition and aggressive high-dose intravenous corticosteroid pulse treatment. J Neurol. 1995;242:326-31. 
34. Moranne O, Hachulla E, Valt AS, Sotoares G, Pagniez D, Boulaner E. Longitudinal Myelitis in a pregnant patient with SLE. Am J Med. 2004;116:355-7.

35. Lehnhardt FG, Holtik U, Burghaus L, Neveling M, Impekoven P, Ruger A, et al. Autologous blood stem cell transplantiation in refractory systemic lupus erythematodes with recurrent longitudinal myelitis and cerebral infarction. Lupus. 2006;15: 240-3.

36. Mok CC, Mak A, To CH. Mycophenolate mofetil for lupus related myelopathy. Ann Rheum Dis. 2006;65:971-3.

37. Saito K, Nawata M, Nakayamada S, Tokunaga M, Tsukada J, Tanaka Y. Successful treatment with anti-CD20 monoclonal antibody (rituximab) of life-threatening refractory systemic lupus erythematosus with renal and central nervous system involvement. Lupus. 2003;12:798-800.

38. Yamanaka F, Migita K, Ichinose K, Ohno N, Kimura H, Ishimaru $\mathrm{H}$, et al. Catastrophic transverse myelitis in a patient with systemic lupus erythematosus. Mod Rheumatol. 2005;15:130-3.

39. Estes D, Christian CL. The natural history of systemic lupus erythematosus by prospective analysis. Medicine (Baltimore). 1971;50:85-95.
40. Grigor R, Edmonds J, Lewkonia R, Bresnihan B, Hughes GR. Systemic lupus erythematosus. A prospective analysis. Ann Rheum Dis. 1978;37:121-8.

41. Abel T, Gladman DD, Urowitz MB. Neuropsychiatric lupus. J Rheumatol. 1980;7:325-33.

42. Omdal R, Selseth B, Kløw NE, Husby G, Mellgren SI. Clinical neurological, electrophysiological, and cerebral CT scan findings in systemic lupus erythematosus. Scand J Rheumatol. 1989;18:283-9.

43. Omdal R, Henriksen OA, Mellren SI, Husby G. Peripheral neuropathy in systemic lupus erythematosus. Neurology. 1991;41:808-11.

44. Omdal R, Løseth S, Torbergsen T, Koldingsnes W, Husby G, Mellgren SI. Peripheral neuropathy in systemic lupus erythematosus--a longitudinal study. Acta Neurol Scand. 2001;103:386-91.

45. Goransson LG, Tjensvoll AB, Herigstad A, Mellgren SI, Omdal R. Small diameter nerve fiber neuropathy in systemic lupus erythematosus. Arch Neurol. 2006;63:401-4. 